\title{
CÁLCULO DO CALOR LATENTE DA LIGA A356.0 PELO MÉTODO DE NEWTON*
}

Gabriel Braga Regattieri Sampaio ${ }^{1}$ Carlos Raimundo Frick Ferreira ${ }^{2}$

\section{Resumo}

Neste trabalho, determinou-se o calor latente de fusão da liga A356.0 através da Curva Zero, calculada pelo método Newtoniano de análise térmica linear. Para tanto, foram feitos experimentos com dois Copos de Análise Térmica (CAT) em moldes cerâmicos. Utilizou-se um sistema de aquisição de dados configurado para obtenção das curvas de resfriamento experimental $(\mathrm{CR})$. Para o processamento matemático da curva de resfriamento e a determinação da Curva Zero Newtoniana (CZN) utilizou-se um programa comercial com planilha de cálculo. Obteve-se os valores de $321 \mathrm{~J} / \mathrm{g}$ e $345 \mathrm{~J} / \mathrm{g}$ para cada copo de análise térmica. Comparando com a bibliografia cujo valor do calor latente é $403 \mathrm{~J} / \mathrm{g}$, a precisão foi de $82 \%$.

Palavras-chave: Alumínio; Análise térmica; Curva de resfriamento; Solidificação.

\section{APPLICATION OF THE NEWTONIAN METHOD TO CALCULATE THE LATENT HEAT OF THE A356.0 ALLOY}

\section{Abstract}

The latent heat of the A356.0 alloy was determined by the zero curve, which is calculated by the Newtonian method of linear thermal analysis. Two experiments were done with cups of thermal analysis in ceramic molds. A data acquisition system was used to capture the cooling curves, and a computer software with calculation spread sheet to determine the Newtonian zero curve. The latent heat calculated in each cup were $321 \mathrm{~J} / \mathrm{g}$ and $345 \mathrm{~J} / \mathrm{g}$. The accuracy was $82 \%$, comparing to the bibliography which latent heat for the same alloy was $403 \mathrm{~J} / \mathrm{g}$.

Keywords: Aluminum; Cooling curve; Thermal analysis; Solidification.

1 Estudante de engenharia metalúrgica, bolsista de iniciação científica, Universidade Federal do Rio Grande do Sul, Porto alegre, Brasil.

2 Engenheiro mecânico, doutor em engenharia, pesquisador, pesquisador da Universidade Federal do Rio Grande do Sul, Porto Alegre, Brasil. 


\section{INTRODUÇÃO}

Neste trabalho foi determinado o calor latente de fusão da liga A356.0 através da Curva Zero, calculada pelo método Newtoniano de análise térmica linear. Ihsan-ulhaq [1] mostra que a curva zero é a derivada primeira de uma curva de resfriamento em que é assumido que o metal não sofre nenhuma mudança de estado. Essa curva não é real, mas é uma forma de se calcular o calor latente com auxílio do computador. A partir dos pontos de temperatura de nucleação e solidus, o programa de computador faz um ajuste linear na derivada primeira da curva de resfriamento. Com esse ajuste, temos a curva zero, e a área entre a derivada da curva de resfriamento e a curva zero nos fornecerá o calor latente. Para tanto, foram feitos dois experimentos com Copos de Análise Térmica (CAT), ambos em moldes cerâmicos. Utilizou-se um sistema de aquisição de dados configurado para obtenção das curvas de resfriamento experimental (CR). Para o processamento matemático da curva de resfriamento e a determinação da curva zero Newtoniana (CZN) utilizouse um programa comercial com planilha de cálculo. $\mathrm{O}$ calor latente $(\mathrm{L})$ foi calculado aplicando a Equação 1.

$\left.L=C_{p} \int_{0}^{t}\left[(d T / d t)_{C R}-(d T / d t)_{C Z N}\right] d t\right]$

Onde $C_{p}$ é o calor específico da liga. Neste caso, foi utilizado o valor $1,3 \mathrm{~J} / \mathrm{g}$ de acordo com [1]. (dT/dt) $)_{\mathrm{CR}}$ corresponde à derivada primeira da curva de resfriamento $\mathrm{e}(\mathrm{dT} / \mathrm{dt})_{\mathrm{cZN}}$ corresponde à derivada primeira da curva zero.

\section{MATERIAIS E MÉTODOS}

No experimento, a liga de alumínio A356 foi vazada em dois copos de análise térmica, instrumentados com um termopar tipo $\mathrm{K}$ em cada, localizados no centro do copo. As medidas de temperatura por tempo foram registradas por um sistema de aquisição de dados e colocados em um software com planilha de cálculo como mostram as figuras 1 e 2 .

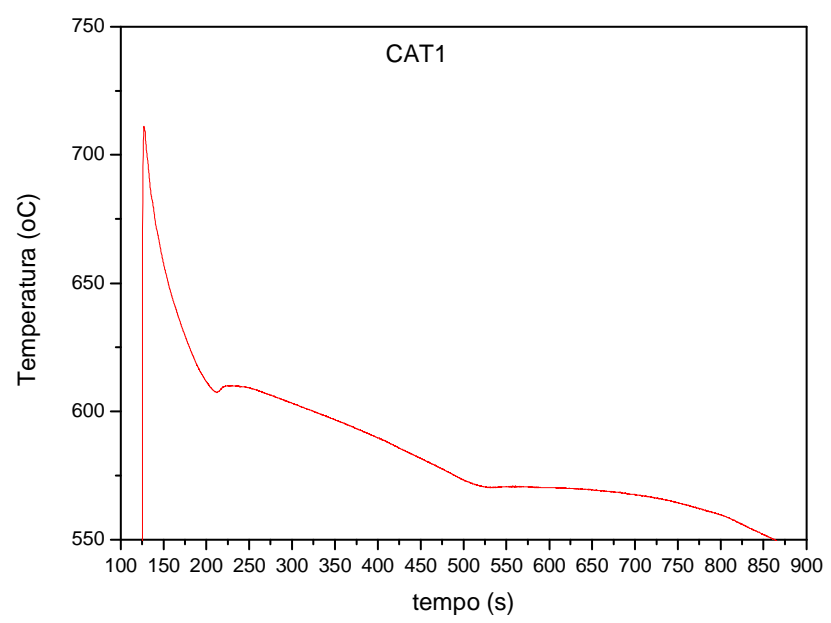

Figura 1. Gráfico da curva de resfriamento do alumínio no copo 1. 


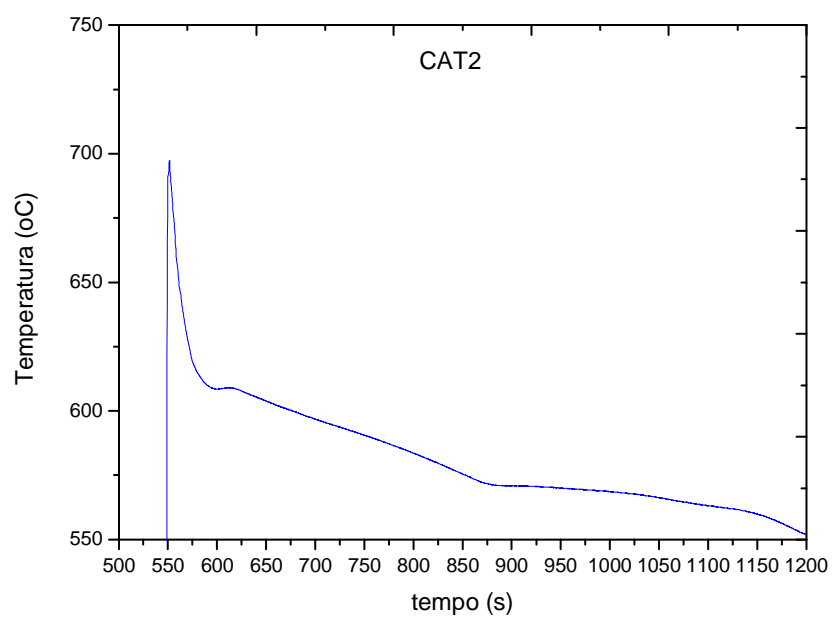

Figura 2. Gráfico da curva de resfriamento do alumínio no copo 2.

Depois de fazer a derivada da curva de resfriamento, determinou-se os pontos de temperatura de nucleação e de temperatura solidus, baseado em Bäckerud et tal [2]. Com auxilio do programa de computador, aplicou-se o ajuste linear na curva da derivada como mostra a figura 3 e 4.

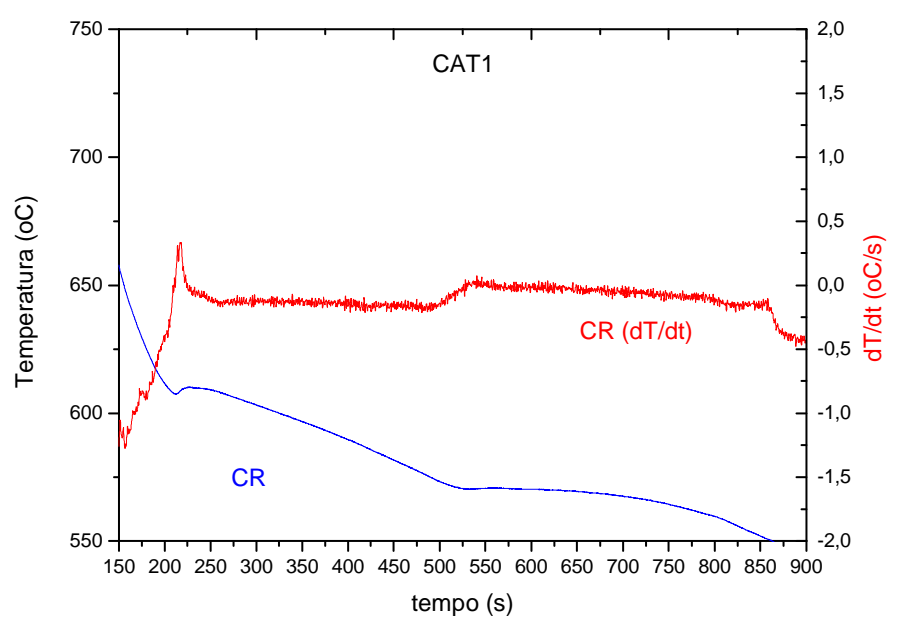

Figura 3. Gráfico com a curva de resfriamento (CR) e a sua derivada primeira.

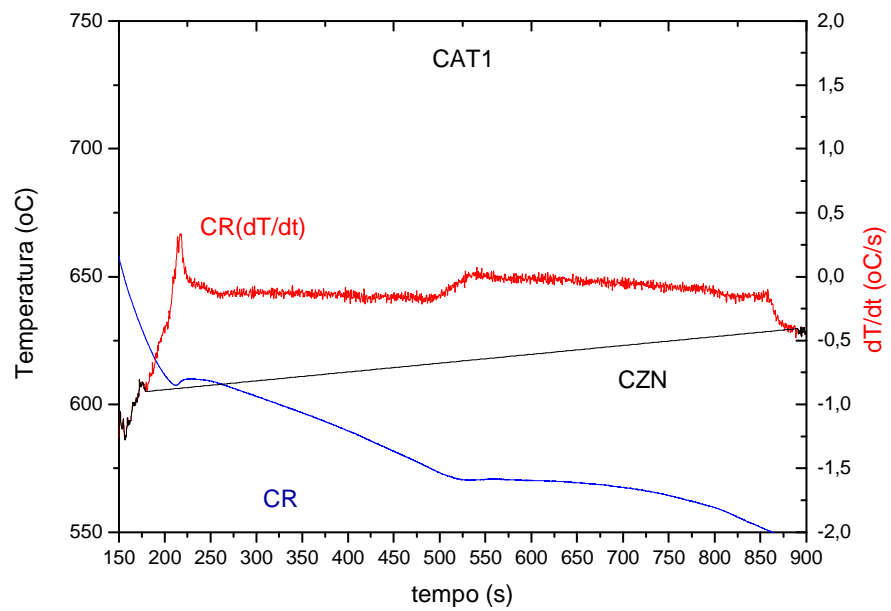

Figura 4. Gráfico com a curva de resfriamento (CR), a sua derivada $1^{\mathrm{a}}$ e a curva zero (CZN) feita com o ajuste linear. 
Com a curva zero (CZN) calculada, o calor latente (L) pode ser obtido com ajuda da Equação 1.

\section{RESULTADOS E DISCUSSÃO}

Os resultados obtidos experimentalmente foram comparados com dados da literatura e estão mostrados na tabela 1

Tabela 1. Resultados do calor latente obtidos experimentalmente e da literatura.

\begin{tabular}{ll}
\hline Experimento & Calor latente, $\mathrm{L}(\mathrm{J} / \mathrm{g})$ \\
\hline CAT1 & 321 \\
CAT2 & 345 \\
Literatura & 403 \\
\hline
\end{tabular}

\subsection{Discussão}

O calor latente da liga A356.0 calculado por Emadi [3] foi de 403J/g. Os valores encontrados experimentalmente apresentam precisão de $82 \%$. Comparados com Ihsan-ul-haq [1] apresentam precisão de $70 \%$.

\section{CONCLUSÃo}

O método newtoniano de análise térmica linear permitiu calcular o calor latente da liga A356. Para trabalhos futuros se estudará o método de Fourier de análise térmica.

\section{Agradecimentos}

Os autores agradecem a FAPERGS por financiar o trabalho, e o primeiro autor agradece ao CNPq pela bolsa de iniciação científica concedida.

\section{REFERÊNCIAS}

1. Haq IU, Shin JS, Lee ZH. Computer-Aided Cooling Curve Analysis of A356 Aluminum Alloy. METALS AND MATERIALS, International. 2004,10(1): 89-96.

2. Bäckerud L, Chai G, Tamminem J. Solidification characteristics of aluminum alloys Vol 2: Foundry Alloys. Stockholm: AFS/SkanAluminum; 1990.

3. Emadi D, Whiting LV, Nafisi S, Ghomashchi R. Applications of Thermal Analysis in Quality Control of Solidification Processes. Journal of Thermal Analysis and Calorimetry. 2005, (81): 235-242. 